\title{
Pseudo Base Location for Mobile Terminal with Abnormal Dynamic Access
}

\author{
Zhu Da-li ${ }^{1,2}$, Shao Jing ${ }^{1,2}$, Jin Hao ${ }^{1,2}$ and Yang Ying ${ }^{1,2}$ \\ ${ }^{1}$ Institute of Information Engineering, Chinese Academy of Sciences, \\ Beijing 100093, China; \\ ${ }^{2}$ University of Chinese Academy of Sciences, Beijing 100049, China \\ E-mail:627955668@qq.com
}

\begin{abstract}
In passive bearing-only localization of onboard mono-station, if abnormal error existed in observed value, using extended Kalman filter (EKF) algorithm can lead to biased results. For enhance the robustness of the algorithm, to construct robustness equivalent gain matrix on the basis of standardized predicted residual, and apply the robustness EKF algorithm to passive bearing-only localization of onboard mono-station. According to the characteristics of inefficient robustness EKF algorithm, combined with $F$ distribution statistics, propose the mono-station passive location algorithm based on the improved extended Kalman filtering, and by adding single abnormal error and continuous abnormal error in observed value, test the resistant ability of algorithm to different abnormal errors. Simulation shows that the proposed algorithm can weaken the influence of abnormal error on position estimation, and can improve the efficiency of positioning based on $F$ distribution discriminant algorithm.
\end{abstract}

Keywords: Passive location; Robustness EKF filtering; $F$ distribution discrimination; Location accuracy

\section{Introduction}

The passive bearing-only localization of onboard mono-station, with strong disguised, good flexibility, has a broad application prospect in the field of electronic warfare, etc. Error! Reference source not found..In some complex environment, angle may be the only observation information can be detected by detection equipment, so the research based on the Angle information of passive bearing-only localization of onboard monostation has important significance.

The frequently-used localization algorithms are least square method, Kalman filtering method, etc. Least square method and its improved algorithm has extensive application in the direction-finding location, such as linear weighted least square method, total least square method, these methods have certain effects on overcome random error obeyed the zero mean gaussian distribution, but when abnormal error existing in observed value, will have great influence on location accuracy. To solve these problems, Literature [2] will introduce robustness estimation theory into the improved algorithm of least square method, thus improve the ability of the algorithm to resist abnormal error, but without considering the state equation of mobile carrier. Considering state equation of system, Kalman filtering algorithm can be used, Literature [4] proposed robustness Kalman filtering, rank-defect model of Kalman filtering, robustness adaptive Kalman filter, and other algorithms, the robustness estimation theory is introduced into the standard Kalman filtering and its improved algorithm. But these algorithms are mostly applied into matching and estimation of satellite clock error currentlyError! Reference source not found., precise point positioning (PPP) Error! Reference source not found., integrated navigationError! Reference source not found., etc. while in respect of passive location 
algorithm, the research on improving the ability of algorithm resistance to abnormal error using robustness estimation principle is less. When applying robustness EKF algorithm into passive bearing-only localization of onboard mono-station, the location efficiency problem needs to be taken into account. To do this, it needs further research on the robustness and effectiveness of localization algorithm.

According to the characteristics of inefficient robustness EKF algorithm, combined with $\mathrm{F}$ distribution statistics, it proposes mono-station passive location algorithm based on the improved extending Kalman filtering, and by adding single abnormal error and continuous abnormal error in observed value, test the resistant ability of algorithm to different abnormal errors. Simulation shows that the proposed algorithm can weaken the influence of abnormal error on position estimation, and can improve the efficiency of positioning based on $\mathrm{F}$ distribution discriminant algorithm.

\section{Passive Bearing-only Localization Model of Onboard Mono-station}

For the convenience of calculation, the passive bearing-only localization model of twodimensional onboard mono-station as an example for analysis, establish the state equation and observation equation of location model, and conduct linearized processing for the observation equation. As shown in figure 1, the target radiation source $T$ is located in $\left(x_{t}, y_{t}\right)$. Let the plane starts from the original point, do uniform rectilinear motion with the speed of $v$, and direction finding, the coordinate of plane at the $k$ th observation point is $\left(x_{k}, y_{k}\right), \phi_{k}$ is the azimuthal angle measured at the $k$ th observation point.

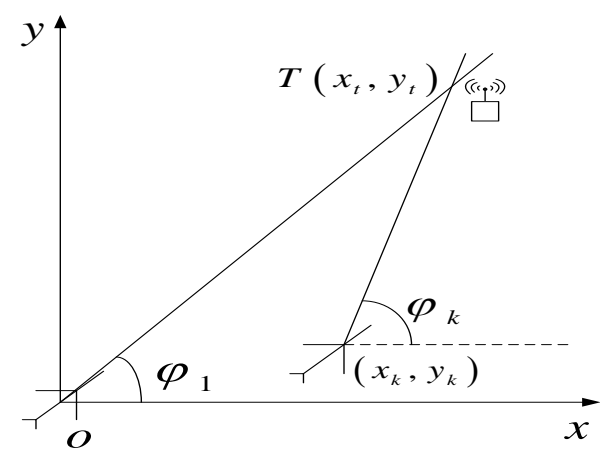

Figure 1. Location Model

With plane as reference system, the state equation of targeted radiation source is:

$$
X(k)=\Phi X(k-1)+\Gamma W(k)
$$

where,

$$
\begin{aligned}
X(k)= & {[x(k), \dot{x}(k), y(k), \dot{y}(k)]^{T} } \\
= & {\left[x_{t}-x_{k},-v_{x}, y_{t}-y_{k},-v_{y}\right]^{T} } \\
\Phi & =\left[\begin{array}{cccc}
1 & T & 0 & 0 \\
0 & 1 & 0 & 0 \\
0 & 0 & 1 & T \\
0 & 0 & 0 & 1
\end{array}\right] \\
\Gamma & =\left[\begin{array}{cc}
T^{2} / 2 & 0 \\
T & 0 \\
0 & T^{2} / 2 \\
0 & T
\end{array}\right]
\end{aligned}
$$


The observation equation of passive bearing-only localization of onboard mono-station can be expressed as:

$$
Z(k)=h[k, X(k)]+V(k)
$$

where,

$$
\begin{gathered}
V(k) \square N(0, R(k)) \\
Z(k)=\left[\phi_{1}, \phi_{2}, \cdots, \phi_{n}\right]^{T} \\
X(k)=\left[x_{t}-x_{k},-v_{x}, y_{t}-y_{k},-v_{y}\right]^{T} h[k, X(k)]=\arctan \left(\frac{y_{t}-y_{k}}{x_{t}-x_{k}}\right)
\end{gathered}
$$

With plane as reference system, location model is converted into fixed mono-station to trace the uniform motion of the target radiation source, which can be solved by using Kalman filtering.

The motion model can be written as:

$$
\begin{gathered}
X(k)=\Phi X(k-1)+\Gamma W(k) \\
Z(k)=\arctan \left(\frac{y_{t}-y_{k}}{x_{t}-x_{k}}\right)+V(k)
\end{gathered}
$$

It is shown in model that the state equation is linear, while observation equation is nonlinear, and linear observation matrix is:

$$
\begin{aligned}
H & =\frac{\partial Z(k)}{\partial X(k)} \\
& =\left[\frac{\partial Z(k)}{\partial x(k)}, \frac{\partial Z(k)}{\partial \dot{x}(k)}, \frac{\partial Z(k)}{\partial y(k)}, \frac{\partial Z(k)}{\partial \dot{y}(k)}\right] \\
& =\left[\frac{-\left(y_{t}-y_{k}\right)^{2}}{\left(x_{t}-x_{k}\right)^{2}+\left(y_{t}-y_{k}\right)^{2}}, 0, \frac{-\left(x_{t}-x_{k}\right)^{2}}{\left(x_{t}-x_{k}\right)^{2}+\left(y_{t}-y_{k}\right)^{2}}, 0\right]
\end{aligned}
$$

The linear approximation of non-linear system can be realized by extending Kalman filtering algorithm, and the location accuracy can be improved. For a given model, its extended Kalman filtering can be further predicted as:

$$
\begin{gathered}
\bar{X}(k+1 \mid k)=\Phi \hat{X}(k \mid k) \\
\bar{Z}(k+1)=h[k+1, \bar{X}(k+1 \mid k)]
\end{gathered}
$$

The predicted covariance matrix is:

$$
P(k+1 \mid k)=\Phi P(k \mid k) \Phi^{T}+Q(k)
$$

Filtering estimated value and its corresponding covariance matrix for:

$$
\begin{aligned}
& \hat{X}(k+1 \mid k+1)= \hat{X}(k+1 \mid k)+ \\
& K(k+1)[Z(k+1)-\hat{Z}(k+1)] \\
& P(k+1 \mid k+1)=[I-K(k+1) H(k+1)] \\
& P(k+1 \mid k)
\end{aligned}
$$

The observation estimated value is:

$$
\hat{Z}(k+1)=h[k+1, \hat{X}(k+1 \mid k+1)]
$$

EKF gain matrix is:

$$
\begin{aligned}
K(k+1)= & P(k+1 \mid k) H^{T}(k+1)[H(k+1) \\
& \left.P(k+1 \mid k) H^{T}(k+1)+R(k+1)\right]^{-1}
\end{aligned}
$$

For the given non-linear location model, the non-linear problem can be solved effectively by using EKF algorithm, and the optimal state estimation can be obtained. 


\section{Robustness Extended Kalman Filtering Algorithm}

\subsection{Robustness EKF Filtering Algorithm}

Assumed the predicted state parameter vector $\hat{X}(k+1 \mid k)$ at $k$ hour obeying the contamination normal distribution, observation vector $Z(k)$ contained abnormal error, and obeyed normal distribution, that is:

$$
Z(k) \square\left(1-\varepsilon_{k}\right) N_{k}+\varepsilon_{k} h_{Z(k)}
$$

Where, $N_{k}$ denotes obeying normal distribution; $h_{Z(k)}$ is contamination source distribution; $\varepsilon_{k}\left(0<\varepsilon_{k}<1\right)$ is contamination rate. The adoptive robustness EKF algorithm, robustness $\mathrm{M}$ estimation is applied on observation vector, while least square estimation is applied on state parameter, that is M-LS filtering.

Robustness EKF process including equivalent gain matrix constructing and iterative solution. The key of robustness estimation is to construct appropriate equivalent weight function, when abnormal observation data appear, weight is reduced and even to 0 , in order to reduce the influence of abnormal data on estimated value. The frequently- used equivalent weight functions in robustness estimation, including Huber weight function, Danish weight function, IGG I weight function, IGGIII weight function, etc. And IGGIII weight function is selected as equivalent weight function, namely:

$$
\bar{p}_{i}= \begin{cases}1 & \left|\tilde{s}_{i}\right| \leq k_{1} \\ \frac{k_{1}}{\mid \tilde{s}_{i}}\left(\frac{k_{2}-\left|\tilde{s}_{i}\right|}{k_{2}-k_{1}}\right)^{2} & k_{1}<\left|\tilde{s}_{i}\right| \leq k_{2} \\ 0 & \left|\tilde{s}_{i}\right|>k_{2}\end{cases}
$$

Where, $\tilde{s}_{i}$ is the standardized residual of $i$ th observation value, $\tilde{s}_{i}=\hat{s}_{i} / \sigma_{v}, \sigma_{v}$ 为 is the standard deviation of residual, can be calculated by transcendental method. $k_{1}$ and $k_{2}$ is constant, which reflects the sensitiveness of algorithm to abnormal data, and $k_{1} \in[1.0,1.5], k_{2} \in[2.5,8.0]$ in general.

State parameter robustness solution $\hat{X}(k+1 \mid k+1)$, its covariance matrix $P(k+1 \mid k+1)$ and robustness EKF gain $K_{M L S}(k+1)$ is :

$$
\begin{aligned}
\hat{X}(k+1 \mid k+1)= & \hat{X}(k+1 \mid k)+K_{\text {MLS }}(k+1) \\
& (Z(k+1)-\hat{Z}(k+1)) \\
P(k+1 \mid k+1)= & {\left[I-K_{\text {MLS }}(k+1)\right.} \\
& H(k+1)] P(k+1 \mid k) \\
K_{M L S}(k+1)= & P(k+1 \mid k) H^{T}(k+1) \\
& {[H(k+1) P(k+1 \mid k)} \\
& \left.H^{T}(k+1)+\bar{R}(k+1)\right]^{-1}
\end{aligned}
$$

Where, $\bar{R}(k+1)=\left[R(k+1) \bar{P}_{k}\right]^{-1}, \bar{P}_{k}$ is equivalent weight matrix; $I$ is unit matrix.

In robustness EKF algorithm, robustness $\mathrm{M}$ estimation is applied for observation vector, while the least square estimation is applied for state parameter. When abnormal error exists in observation value, the algorithm can reduce the weight on abnormal data through robustness equivalent gain matrix, so as to reduce the influence of abnormal data on estimated location value. 


\subsection{Robustness EKF Algorithm based on F Distribution Discrimination}

Error equation of state predictive information vector:

$$
V_{\bar{X}_{k}}(k)=\hat{X}(k \mid k)-\bar{X}(k \mid k-1)
$$

And statistics Error! Reference source not found. :

$$
e(k)=\left(V_{\bar{X}_{k}}^{T}(k)\right)(P(k \mid k-1))^{-1}\left(V_{\bar{X}_{k}}(k)\right)
$$

Obey $\chi^{2}$ distribution with degree of freedom of 4 .

Error equation of observation vector:

$$
V(k)=Z(k)-\hat{Z}(k)
$$

And statistics:

$$
h(k)=\left(V^{T}(k)\right)(P(k \mid k))^{-1}(V(k))
$$

Obey $\chi^{2}$ distribution with degree of freedom of 1 .

Construct statistics:

$$
F(k)=\frac{e(k)}{4} / \frac{h(k)}{1}
$$

When no abnormal error existing in observed value and state predicted value, the observation residual and state error follow zero mean normal distribution, statistics $F(k)$ obeys central $F$ distribution with freedom degrees of $(4,1)$; when abnormal error existing in observed value and state predicted value, the observation residual and state error follow non-zero mean normal distribution, statistics $F(k)$ obeys non-central $F$ distribution with freedom degrees of $(4,1)$.

Test method:

(1) State error obeys zero-mean normal distribution, and abnormal error existing in observed value.

Original hypothesis $H_{0}$ : no abnormal error existing observed value; alternative hypothesis $H_{1}$ : abnormal error existing observed value. Significance level is $\alpha$, if $F(k)>F_{\alpha}(4,1)$, then $H_{0}$ hypothesis is accepted, test is passed; otherwise, $H_{1}$ hypothesis is accepted, $H_{0}$ hypothesis is rejected. Where, $F_{\alpha}(4,1)$ is the $\alpha$ quantile on $F(4,1)$ distribution.

(2) observed residual obeys zero-mean normal distribution, and abnormal error existing in state predicted value.

Original hypothesis $H_{0}$ : no abnormal error existing observed value; alternative hypothesis $H_{1}$ : abnormal error existing observed value. Significance level is $\alpha$, if $F(k) \leq F_{\alpha}(4,1)$, then $H_{0}$ hypothesis is accepted, test is passed; otherwise, $H_{1}$ hypothesis is accepted, $H_{0}$ hypothesis is rejected.

This paper only consider the condition of abnormal error existing in observed value, therefore, before robustness iteration, it can be tested with above mentioned method first, if there is abnormal error, proceed robustness iteration, and get accurate estimated location value; if there is no abnormal error, no longer proceed robustness iteration, and the estimated location value obtained by EKF algorithm as the estimated result.

\section{Simulated Analysis}

\subsection{Simulated Parameter Setting}

Simulation parameter settings are as follows: a radiation source target $T$ locates at (5 $\mathrm{km}, 10 \mathrm{~km})$, the plane starts from $(0,0)$ at a speed of $600 \mathrm{~m} / \mathrm{s}$, moves toward the $x$ positive half axis and direction finding, measurement cycle is $1 \mathrm{~s}$, measurement noise 
obeys normal distribution with mean value of 0 , variance of $0.1^{\circ}$. In order to speed up the location speed and ensure the convergence of robustness EKF algorithm, set recursive estimated initial value of target location calculated by the previous measured values. $\boldsymbol{P}$ matrix initial value is $\boldsymbol{P}(0)=\operatorname{diag}\left(10^{6}, 0,10^{6}, 0\right)$, with the plane as reference system, the target speed is known, so the initial variance of speed is set to 0. In improved EKF algorithm, the significance level $\alpha=0.005$, it can be obtain from table look-up, the judgment threshold $F_{\alpha}(4,1)=22500$. In order to analyze algorithm performance accurately, carrying out Monte-Carlo simulation for many times, using RMSE performance indexes, namely

$$
\text { RMSE }=\sqrt{\frac{1}{M} \sum_{i=1}^{M}\left[\left(x_{t}-x_{k}-\hat{x}_{i}\right)^{2}+\left(y_{t}-y_{k}-\hat{y}_{i}\right)^{2}\right]}
$$

Where, $M$ is the Monte-Carlo simulation times, and $M=200$ in this paper. Set simulation time is 200 s.

\subsection{Results and Analysis}

4.2.1. Performance Comparison of EKF Algorithm and Least-square Method: Performance comparison of EKF algorithm and least square method is shown in figure 2. It can be seen from the figure 2 that, location accuracy and convergence speed of EKF algorithm are better than those of least square method, after convergence, the difference of location accuracy of both is around $150 \mathrm{~m}$. This is because the EKF algorithm considering the state equation of system, and can obtain more information, therefore can get more accurate estimated location value.

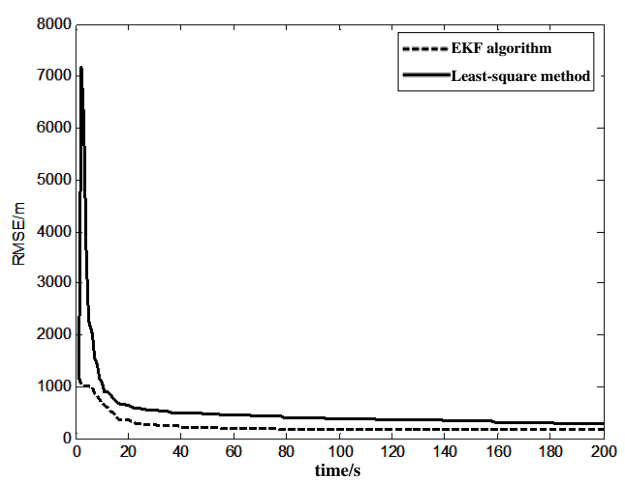

\section{Figure 2. Performance Comparison of EKF Algorithm and Least-square Method}

4.2.2. Performance Comparison at No Abnormal Error Existing: When no abnormal error existing, the performance comparison of EKF algorithm, robustness EKF algorithm and robustness EKF algorithm based on $\mathrm{F}$ distribution discrimination, as shown in figure 3. It can be seen from figure 3 that, when no abnormal error existing, with the increase of observation time, EKF algorithm and robustness EKF algorithm are convergent gradually. In particular, convergence speed of robustness EKF filtering algorithm is slower than that of EKF algorithm, and the location accuracy is lower than the EKF algorithm after convergence, the location accuracy difference of two algorithms is about $20 \mathrm{~m}$. This is due to that, the robustness EKF algorithm conducts robustness iteration on the observation value at every moment, which makes the calculating time is longer and the location accuracy is reduced, the location accuracy differences about $20 \mathrm{~m}$. And EKF algorithm based on $\mathrm{F}$ distribution discriminant and $\mathrm{EKF}$ algorithm has similar performance, the convergence speed and precision are better than the poor resistance to EKF algorithm, the location accuracy difference of two algorithms is about $3 \mathrm{~m}$. Thus it can be seen that, if no 
abnormal error existing, compared with robustness EKF algorithm, the robustness EKF algorithm based on $\mathrm{F}$ distribution discriminant can improve the location accuracy and convergence speed.

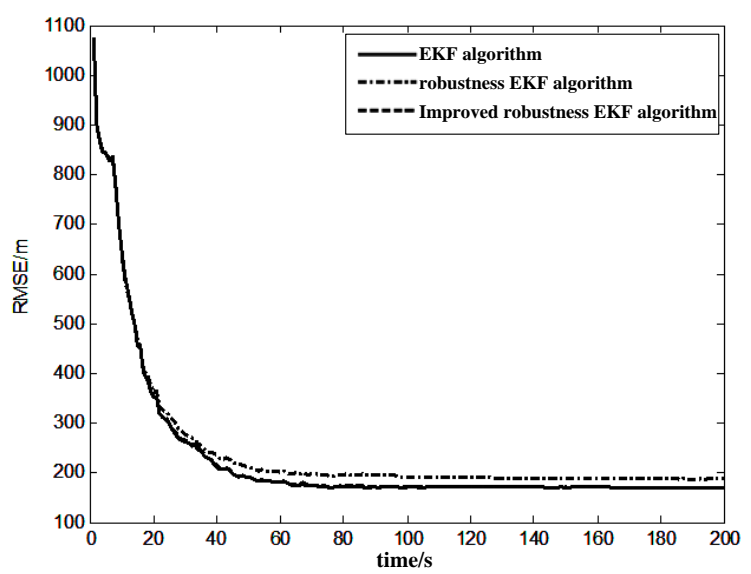

Figure 3. Performance Comparison of Different Algorithms When No Abnormal Error Existing

4.2.3. Performance Comparison when Abnormal Error Existing: Add single abnormal error and continuous abnormal error in observation value, the abnormal error settings as shown in table 1, the performance comparison of EKF algorithm, robustness EKF algorithm and robustness EKF algorithm based on $\mathrm{F}$ distribution discrimination, as shown in figure 4 . It can be seen from figure 4 that, when no abnormal error adding, the performance of robustness EKF algorithm based on $\mathrm{F}$ distribution discrimination is similar to that of EKF algorithm, the performance of both is superior to that of robustness EKF algorithm; when adding continuous abnormal error at $T=100 \square 110 s$, the EKF algorithm is affected by abnormal error, the convergence speed of algorithm is slow down, while robustness EKF algorithm and robustness EKF algorithm based on F distribution discrimination are less been affected, and the location accuracy difference of common algorithm and robustness algorithm is about $100 \mathrm{~m}$. When adding single abnormal error at $T=130,140,160 s$ 处, respectively, the EKF algorithm is affected by abnormal error, location accuracy is reduced, convergence speed is slow, and compared with robustness EKF algorithm and robustness EKF algorithm based on $\mathrm{F}$ distribution discrimination, the location accuracy difference is up to hundreds of meters, especially, adding large abnormal error at $T=160 s$, the EKF algorithm is greatly affected, and seriously distorted, while robustness EKF algorithm and robustness EKF algorithm based on $\mathrm{F}$ distribution discrimination have carried on robustness iteration to abnormal data, namely to reducing weight of abnormal observed value or eliminate it, reduce its influence on estimated location value, so the algorithm can have better convergence, and higher location accuracy. On the whole, the robustness algorithm can better weaken the effect of abnormal error to estimated location value, and guarantee the performance of the algorithm; while robustness EKF algorithm based on $\mathrm{F}$ distribution discrimination is superior to robustness EKF algorithm, this is due to robustness EKF algorithm based on $\mathrm{F}$ distribution discrimination combined with statistical model, constructs the statistical inspection amount obeyed $\mathrm{F}$ distribution and makes judgment, to improve the efficiency of the algorithm and makes the algorithm has faster convergence speed and higher location accuracy. 
Table 1. Abnormal Error Settings

\begin{tabular}{ccccc}
\hline observation point & $100^{\sim} 110 \mathrm{~s}$ & $130 \mathrm{~s}$ & $140 \mathrm{~s}$ & $160 \mathrm{~s}$ \\
\hline standard deviation of abnormal error setting & $5 \sigma$ & $10 \sigma$ & $20 \sigma$ & $25 \sigma$ \\
\hline
\end{tabular}

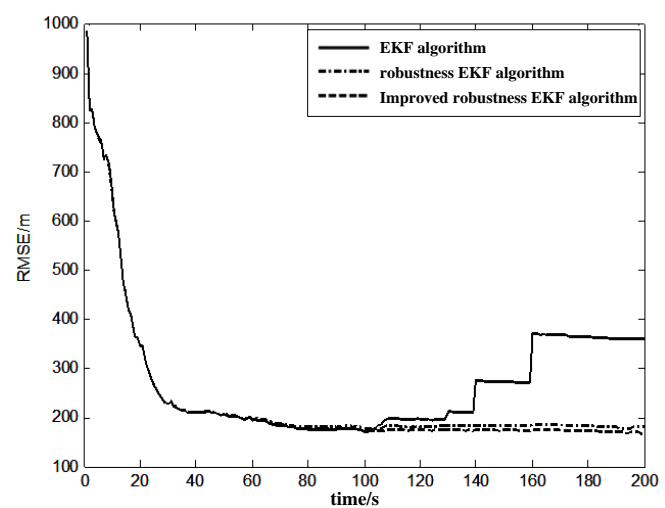

Figure 4. Performance Comparison of Different Algorithms when Abnormal Error Existing

\section{Conclusion}

In this paper, the robustness EKF algorithm is applied in passive bearing-only localization of onboard mono-station, and the robustness EKF algorithm based on $\mathrm{F}$ distribution discrimination is proposed. Simulation shows that this algorithm can resist the effect of abnormal error, with better location performance. Under the condition of significant abnormal existing between state equation and carrier motion trail, and both observation equation and state equation are affected by abnormal error, the estimated value of passive location will also be affected, and produces deviation, these complex environment will be considered in the next step, and the robustness of the algorithm will be further improved.

\section{Acknowledgement}

The National High Technology Research and Development Program of China (863 Program) (2012AA013104)

\section{References}

[1] T. Su, W. Wang and Z. Lv, "Rapid Delaunay triangulation for randomly distributed point cloud data using adaptive Hilbert curve", Computers \& Graphics, (2016), vol. 54, pp. 65-74.

[2] J. Hu, Z. Gao and W. Pan, "Multiangle Social Network Recommendation Algorithms and Similarity Network Evaluation", Journal of Applied Mathematics, 2013, (2013).

[3] S. Zhou, L. Mi, H. Chen and Y. Geng, "Building detection in Digital surface model", 2013 IEEE International Conference on Imaging Systems and Techniques (IST), (2012).

[4] J. He, Y. Geng and K. Pahlavan, "Toward Accurate Human Tracking: Modeling Time-of-Arrival for Wireless Wearable Sensors in Multipath Environment”, IEEE Sensor Journal, vol. 14, no. 11, (2014), pp. 3996-4006.

[5] Z. Lv, A. Halawani and S. Fen, "Touch-less Interactive Augmented Reality Game on Vision Based Wearable Device", Personal and Ubiquitous Computing, vol. 19, no. 3, (2015), pp. 551-567.

[6] G. Bao, L. Mi, Y. Geng, M. Zhou and K. Pahlavan, "A video-based speed estimation technique for localizing the wireless capsule endoscope inside gastrointestinal tract", 2014 36th Annual International Conference of the IEEE Engineering in Medicine and Biology Society (EMBC), (2014).

[7] D. Zeng and Y. Geng, "Content distribution mechanism in mobile P2P network", Journal of Networks, vol. 9, no. 5, (2014), pp. 1229-1236. 
[8] W. Gu, Z. Lv and M. Hao, "Change detection method for remote sensing images based on an improved Markov random field", Multimedia Tools and Applications, (2015), pp. 1-16.

[9] Z. Chen, W. Huang and Z. Lv, "Towards a face recognition method based on uncorrelated discriminant sparse preserving projection", Multimedia Tools and Applications, (2015), pp. 1-15.

[10] J. Hu and Z. Gao, "Distinction immune genes of hepatitis-induced heptatocellular carcinoma", Bioinformatics, vol. 28, no. 24, (2012), pp. 3191-3194

[11] T. Su, W. Wang and Z. Lv, "Rapid Delaunay triangulation for randomly distributed point cloud data using adaptive Hilbert curve", Computers \& Graphics, vol. 54, (2016), pp. 65-74.

[12] W. Gu, Z. Lv and M. Hao, "Change detection method for remote sensing images based on an improved Markov random field", Multimedia Tools and Applications, (2015), pp. 1-16.

[13] Z. Lv, A. Tek and F. Da Silva, "Game on, science-how video game technology may help biologists tackle visualization challenges", PloS one, vol. 8, no. 3, (2013), 57990.

[14] Z. Chen, W. Huang and Z. Lv, "Towards a face recognition method based on uncorrelated discriminant sparse preserving projection", Multimedia Tools and Applications, (2015), pp. 1-15.

[15] D. Jiang, X. Ying and Y. Han, "Collaborative multi-hop routing in cognitive wireless networks", Wireless Personal Communications, (2015), pp. 1-23.

[16] Z. Lv, A. Tek and F. Da Silva, "Game on, science-how video game technology may help biologists tackle visualization challenges", PloS one, vol. 8, no. 3, (2013), 57990.

[17] D. Jiang, Z. Xu and Z. Lv, "A multicast delivery approach with minimum energy consumption for wireless multi-hop networks", Telecommunication Systems, (2015), pp. 1-12.

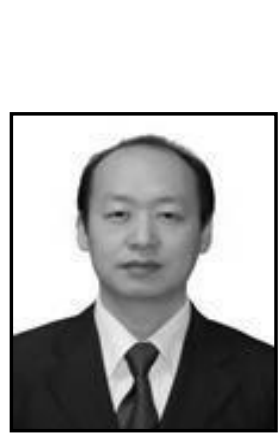

\section{Authors}

Zhu Dal, he received his D.E. degree from Huazhong University of Science and Technology in Wuhan, China. He is currently a senior engineer in the Institute of Information Engineering, Chinese Academy of Sciences. His research interest is mainly in the area of mobile information security, wireless network attack and defense technology. He has published several research papers in scholarly journals and conferences in the above research areas.

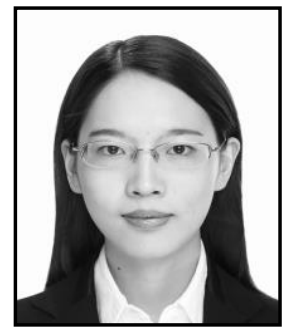

Shao Jing, she received her BE degree in Information Engineering from Beijing Institute of Technology in Beijing, China. She is currently a master in the Institute of Information Engineering, Chinese Academy of Sciences. Her research interest is mainly in mobile information security, the control of mobile phone based on base station.

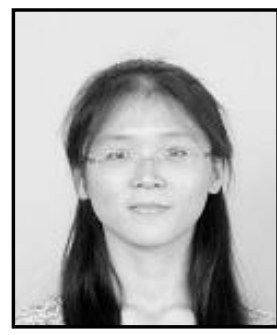

Ji Hao, she received her BE Degree in computer science and technology from Hefei University of Technology in Anhui, China. She is currently a master student in the Institute of Information Engineering, Chinese Academy of Sciences. Her research interest is mainly in the area of mobile information security and the detection technology of mobile malwares.

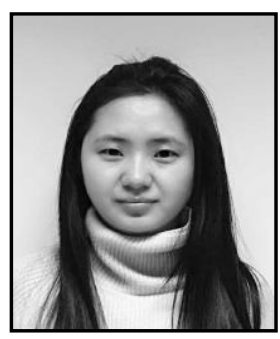

Yang Ying, she received her ME Degree in computer science and technology from Henan University, China. She is currently a doctor student in the Institute of Information Engineering, Chinese Academy of Sciences. Her research interest is mainly in the area of mobile information security and operating system security. 
International Journal of Future Generation Communication and Networking Vol. 9, No. 11 (2016) 